Article

\title{
New Applications of the Fractional Integral on Analytic Functions
}

\author{
Alina Alb Lupaş \\ Department of Mathematics and Computer Science, University of Oradea, 1 Universitatii Street, \\ 410087 Oradea, Romania; dalb@uoradea.ro or alblupas@gmail.com
} iations.

\begin{abstract}
The fractional integral is a function known for the elegant results obtained when introducing new operators; it has proved to have interesting applications. In the present paper, differential subordinations and superodinations for the fractional integral of the confluent hypergeometric function introduced in a previously published paper are presented. A sandwich-type theorem at the end of the original part of the paper connects the outcomes of the studies done using the dual theories.
\end{abstract}

Keywords: differential subordination; differential superordination; fractional integral; confluent hypergeometric function

\section{Introduction}

It is a known fact that the notion of an operator was used from the early stage of the study of complex-valued functions; many already known results can be proven easier with them, and new results are being obtained with them.

Many papers, such as [1,2], studied different operators defined by using the fractional integral of order $\lambda$ also used earlier by S. Owa [3]. Despite that, we also refer to [4-6] for theoretical and numerical analyses from real models described by classical PDEs and related operators. The results contained in the present paper were inspired by the outstanding results previously obtained using fractional integrals, and the study was done by applying them to a confluent hypergeometric function. The definition of a fractional integral can be seen in [3] as follows:

Definition 1 ([3]). The fractional integral of order $\alpha$ is defined by

$$
D_{z}^{-\alpha} f(z)=\frac{1}{\Gamma(\alpha)} \int_{0}^{z} \frac{f(\zeta)}{(\zeta-z)^{1-\alpha}} d \zeta
$$

where $\alpha$ is a positive real number, $f(z)$ is an analytic function in a simply connected region of the $z$-plane containing the origin and the multiplicity of $(\zeta-z)^{\alpha-1}$ is removed by requiring $\ln (\zeta-z)$ to be real when $(\zeta-z)>0$.

In paper [7] a new operator was introduced by using a fractional integral on the confluent (Kummer) hypergeometric function. The introduction of this operator was inspired by the studies done on this function having in view many aspects, from its combination with other functions, as can be seen in papers [8,9], to its univalence in paper [10].

The confluent (Kummer) hypergeometric function of the first kind is defined in [11] as follows:

Definition 2 ([11]). Let $a, c \in \mathbb{C}, c \neq 0,-1,-2, \ldots$ and consider

$$
\phi(a, c ; z)={ }_{1} F_{1}(a, c ; z)=1+\frac{a}{c} \frac{z}{1 !}+\frac{a(a+1)}{c(c+1)} \frac{z^{2}}{2 !}+\ldots, z \in U .
$$


This function is called a confluent (Kummer) hypergeometric function, is analytic in $\mathbb{C}$ and satisfies Kummer's differential equation:

$$
z w^{\prime \prime}(z)+(c-z) w^{\prime}(z)-a w(z)=0
$$

Considering

$$
(d)_{k}=\frac{\Gamma(d+k)}{\Gamma(d)}=d(d+1)(d+2) \ldots(d+k-1) \text { with }(d)_{0}=1,
$$

the confluent (Kummer) hypergeometric function can be written as

$$
\phi(a, c ; z)=\sum_{k=0}^{\infty} \frac{(a)_{k}}{(c)_{k}} \frac{z^{k}}{k !}=\frac{\Gamma(c)}{\Gamma(a)} \sum_{k=0}^{\infty} \frac{\Gamma(a+k)}{\Gamma(c+k)} \frac{z^{k}}{k !} .
$$

The definition of the operator introduced in [7] is the following:

$$
\begin{gathered}
D_{z}^{-\lambda} \phi(a, c ; z)=\frac{1}{\Gamma(\lambda)} \int_{0}^{z} \frac{\phi(a, c ; t)}{(z-t)^{1-\lambda}} d t= \\
\frac{1}{\Gamma(\lambda)} \frac{\Gamma(c)}{\Gamma(a)} \sum_{k=0}^{\infty} \frac{\Gamma(a+k)}{\Gamma(c+k) \Gamma(k+1)} \int_{0}^{z} \frac{t^{k}}{(z-t)^{1-\lambda}} d t,
\end{gathered}
$$

where $\lambda>0, a, c \in \mathbb{C}, c \neq 0,-1,-2, \ldots$

The fractional integral of a confluent hypergeometric function can be written as

$$
D_{z}^{-\lambda} \phi(a, c ; z)=\frac{\Gamma(c)}{\Gamma(a)} \sum_{k=0}^{\infty} \frac{\Gamma(a+k)}{\Gamma(c+k) \Gamma(\lambda+k+1)} z^{k+\lambda},
$$

after a simple calculation. Evidently, $D_{z}^{-\lambda} \phi(a, c ; z) \in \mathcal{H}[0, \lambda]$.

The original results which are shown in the next part of this paper were obtained by using this operator and differential subordination and superodination theories, synthesized in the monography [12] published by Miller and Mocanu in 2000 and in paper [13], respectively. The usual notion and definitions are considered.

$U=\{z \in \mathbb{C}:|z|<1\}$ is the unit disc of the complex plane, $\mathcal{H}(U)$ the class of analytic functions in $U$ and $\mathcal{H}[a, n]=\left\{f \in \mathcal{H}(U): f(z)=a+a_{n} z^{n}+a_{n+1} z^{n+1}+\ldots, z \in U\right\}$, with $n$ a positive integer and $a \in \mathbb{C}$.

Definition 3 ([12]). Let $f, F \in \mathcal{H}(U)$. The function $f$ is said to be subordinate to $F$ if there exists a Schwarz function $w$, analytic in $U$, with $w(0)=0$ and $|w(z)|<1, z \in U$, such that $f(z)=F(w(z)), z \in U$. In such a case, we write $f \prec F$. If $F$ is univalent, then $f \prec F$ if and only if $f(0)=g(0)$ and $f(U) \subset g(U)$.

Definition 4 ([12]). Let $\psi: \mathbb{C}^{3} \times U \rightarrow \mathbb{C}$ and let $h$ be univalent in $U$. If $p$ is analytic and satisfies the differential subordination

$$
\psi\left(p(z), z p^{\prime}(z), z^{2} p^{\prime \prime}(z) ; z\right) \prec h(z), z \in U,
$$

then $p$ is called a solution of the differential subordination. The univalent function $q$ is called $a$ dominant of the solutions of the differential subordination, or more simply a dominant, if $p \prec q$ for all $p$ satisfying (5). A dominant $\widetilde{q}$ that satisfies $\widetilde{q} \prec q$ for all dominants $q$ of (5) is said to be the best dominant of (5).

The notion related to differential superordinations was introduced in [13]. 
Definition 5 ([13]). Let $\varphi: \mathbb{C}^{3} \times \bar{U} \rightarrow \mathbb{C}$ and let $h$ be analytic in $U$. If $p$ and $\varphi\left(p(z), z p^{\prime}(z)\right.$, $\left.z^{2} p^{\prime \prime}(z) ; z\right)$ are univalent in $U$ and satisfy the differential superordination

$$
h(z) \prec \varphi\left(p(z), z p^{\prime}(z), z^{2} p^{\prime \prime}(z) ; z\right), \quad z \in U,
$$

then $p$ is called a solution of the differential superordination (6). An analytic function $q$ is called a subordinant of the solutions of the differential superordination or more simply a subordinant, if $q \prec p$ for all $p$ satisfying (6). A subordinant $\widetilde{q}$ that satisfies $q \prec \widetilde{q}$ for all subordinants $q$ of (6) is said to be the best subordinant of (6).

In the process of obtaining the original results from this paper, the following lemmas are needed:

Lemma 1 ([12]). Let the function $q$ be univalent in the unit disc $U$ and $\theta$ and $\phi$ be analytic in a domain D containing $q(U)$ with $\phi(w) \neq 0$ when $w \in q(U)$. Set $Q(z)=z q^{\prime}(z) \phi(q(z))$ and $h(z)=\theta(q(z))+Q(z)$. Suppose that $Q$ is star-like univalent in $U$ and $\operatorname{Re}\left(\frac{z h^{\prime}(z)}{Q(z)}\right)>0, z \in U$.

If $p$ is analytic with $p(0)=q(0), p(U) \subseteq D$ and

$$
\theta(p(z))+z p^{\prime}(z) \phi(p(z)) \prec \theta(q(z))+z q^{\prime}(z) \phi(q(z)),
$$

then $p(z) \prec q(z)$ and $q$ is the best dominant.

Lemma 2 ([14]). Let the function $q$ be convex univalent in the open unit disc $U$ and $v$ and $\phi$ be analytic in a domain $D$ containing $q(U)$. Suppose that $\operatorname{Re}\left(\frac{v^{\prime}(q(z))}{\phi(q(z))}\right)>0, z \in U$ and $\psi(z)=z q^{\prime}(z) \phi(q(z))$ is star-like univalent in $U$. $U$ and

If $p(z) \in \mathcal{H}[q(0), 1] \cap Q$, with $p(U) \subseteq D$ and $v(p(z))+z p^{\prime}(z) \phi(p(z))$ is univalent in

$$
v(q(z))+z q^{\prime}(z) \phi(q(z)) \prec v(p(z))+z p^{\prime}(z) \phi(p(z)),
$$

then $q(z) \prec p(z)$ and $q$ is the best subordinant.

\section{Main Results}

Continuing the work from [7], we get:

Theorem 1. Let $q$ be an analytic and univalent function in $U$ with $q(z) \neq 0, \forall z \in U$ and $\frac{z\left(D_{z}^{-\lambda} \phi(a, c ; z)\right)^{\prime}}{D_{z}^{-\lambda} \phi(a, c ; z)} \in \mathcal{H}(U)$, where $\lambda>0$ and $a, c \in \mathbb{C}, c \neq 0,-1,-2, \ldots$ Suppose that $\frac{z q^{\prime}(z)}{q(z)}$ is star-like univalent in $U$. Consider

$$
\operatorname{Re}\left(\frac{2 \mu}{\beta} q^{2}(z)+\frac{\xi}{\beta} q(z)+1-z \frac{q^{\prime}(z)}{q(z)}+z \frac{q^{\prime \prime}(z)}{q^{\prime}(z)}\right)>0, z \in U,
$$

with $\mu, \beta, \xi, \alpha \in \mathbb{C}, \beta \neq 0$, and

$$
\begin{gathered}
\psi_{\lambda}^{a, b}(\alpha, \beta, \xi, \mu ; z):=\alpha+\beta+(\xi-\beta) \frac{z\left(D_{z}^{-\lambda} \phi(a, c ; z)\right)^{\prime}}{D_{z}^{-\lambda} \phi(a, c ; z)}+ \\
\mu\left(\frac{z\left(D_{z}^{-\lambda} \phi(a, c ; z)\right)^{\prime}}{D_{z}^{-\lambda} \phi(a, c ; z)}\right)^{2}+\beta \frac{z\left(D_{z}^{-\lambda} \phi(a, c ; z)\right)^{\prime \prime}}{\left(D_{z}^{-\lambda} \phi(a, c ; z)\right)^{\prime}} .
\end{gathered}
$$

If the following subordination is satisfied by $q$,

$$
\psi_{\lambda}^{a, b}(\alpha, \beta, \xi, \mu ; z) \prec \alpha+\xi q(z)+\mu(q(z))^{2}+\beta \frac{z q^{\prime}(z)}{q(z)},
$$


then

$$
\frac{z\left(D_{z}^{-\lambda} \phi(a, c ; z)\right)^{\prime}}{D_{z}^{-\lambda} \phi(a, c ; z)} \prec q(z),
$$

and $q$ is the best dominant.

Proof. Consider

$$
p(z):=\frac{z\left(D_{z}^{-\lambda} \phi(a, c ; z)\right)^{\prime}}{D_{z}^{-\lambda} \phi(a, c ; z)}, z \in U, z \neq 0 .
$$

By differentiating with respect to $z$, we get

$$
\begin{aligned}
p^{\prime}(z)=\frac{\left(D_{z}^{-\lambda} \phi(a, c ; z)\right)^{\prime}}{D_{z}^{-\lambda} \phi(a, c ; z)}+z \frac{\left(D_{z}^{-\lambda} \phi(a, c ; z)\right)^{\prime \prime}}{D_{z}^{-\lambda} \phi(a, c ; z)}-z\left(\frac{\left(D_{z}^{-\lambda} \phi(a, c ; z)\right)^{\prime}}{D_{z}^{-\lambda} \phi(a, c ; z)}\right)^{2} \text { and } \\
\frac{z p^{\prime}(z)}{p(z)}=1-z \frac{\left(D_{z}^{-\lambda} \phi(a, c ; z)\right)^{\prime}}{D_{z}^{-\lambda} \phi(a, c ; z)}+z \frac{\left(D_{z}^{-\lambda} \phi(a, c ; z)\right)^{\prime \prime}}{\left(D_{z}^{-\lambda} \phi(a, c ; z)\right)^{\prime}} .
\end{aligned}
$$

By setting

$$
\phi(w):=\frac{\beta}{w}
$$

and

$$
\theta(w):=\alpha+\xi w+\mu w^{2},
$$

evidently $\phi$ is analytic in $\mathbb{C} \backslash\{0\}, \phi(w) \neq 0, \forall w \in \mathbb{C} \backslash\{0\}$ and $\theta$ is analytic in $\mathbb{C}$.

Considering

$$
Q(z)=z q^{\prime}(z) \phi(q(z))=\beta \frac{z q^{\prime}(z)}{q(z)}
$$

and

$$
h(z)=\theta(q(z))+Q(z)=\alpha+\xi q(z)+\mu(q(z))^{2}+\beta \frac{z q^{\prime}(z)}{q(z)},
$$

which reveals that $Q$ is a star-like univalent function in $U$.

By differentiating, we obtain $h^{\prime}(z)=\xi q^{\prime}(z)+2 \mu q(z) q^{\prime}(z)+\beta \frac{q^{\prime}(z)}{q(z)}+\beta z \frac{q^{\prime \prime}(z)}{q(z)}-\beta z\left(\frac{q^{\prime}(z)}{q(z)}\right)^{2}$ and $\frac{z h^{\prime}(z)}{Q(z)}=\frac{2 \mu}{\beta} q^{2}(z)+\frac{\xi}{\beta} q(z)+1-z \frac{q^{\prime}(z)}{q(z)}+z \frac{q^{\prime \prime}(z)}{q(z)}$.

We deduce that $\operatorname{Re}\left(\frac{z h^{\prime}(z)}{Q(z)}\right)=\operatorname{Re}\left(\frac{2 \mu}{\beta} q^{2}(z)+\frac{\tilde{\xi}}{\beta} q(z)+1-z \frac{q^{\prime}(z)}{q(z)}+z \frac{q^{\prime \prime}(z)}{q(z)}\right)>0$.

By using (11), we obtain $\alpha+\xi p(z)+\mu(p(z))^{2}+\beta \frac{z p^{\prime}(z)}{p(z)}=$ $\alpha+\beta+(\xi-\beta) \frac{z\left(D_{z}^{-\lambda} \phi(a, c ; z)\right)^{\prime}}{D_{z}^{-\lambda} \phi(a, c ; z)}+\mu\left(\frac{z\left(D_{z}^{-\lambda} \phi(a, c ; z)\right)^{\prime}}{D_{z}^{-\lambda} \phi(a, c ; z)}\right)^{2}+\beta \frac{z\left(D_{z}^{-\lambda} \phi(a, c ; z)\right)^{\prime \prime}}{\left(D_{z}^{-\lambda} \phi(a, c ; z)\right)^{\prime}}$.

By using (9), we have $\alpha+\xi p(z)+\mu(p(z))^{2}+\beta \frac{z p^{\prime}(z)}{p(z)} \prec \alpha+\xi q(z)+\mu(q(z))^{2}+\beta \frac{z q^{\prime}(z)}{q(z)}$.

By applying Lemma 1 , we get $p(z) \prec q(z), \forall z \in U$, which means $\frac{z\left(D_{z}^{-\lambda} \phi(a, c ; z)\right)^{\prime}}{D_{z}^{-\lambda} \phi(a, c ; z)} \prec q(z)$, $\forall z \in U$ and the function $q$ is the best dominant.

Corollary 1. Let $\lambda>0, a, c, \alpha, \beta, \xi, \mu \in \mathbb{C}, c \neq 0,-1,-2, \ldots, \beta \neq 0$, and relation (7) holds for $q(z)=\frac{1+A z}{1+B z}$, with $-1 \leq B<A \leq 1$. If

$$
\psi_{\lambda}^{a, b}(\alpha, \beta, \xi, \mu ; z) \prec \alpha+\xi \frac{1+A z}{1+B z}+\mu\left(\frac{1+A z}{1+B z}\right)^{2}+\frac{\beta(A-B) z}{(1+A z)(1+B z)},
$$

with $\psi_{\lambda}^{a, b}$, which is defined in (8), then

$$
\frac{z\left(D_{z}^{-\lambda} \phi(a, c ; z)\right)^{\prime}}{D_{z}^{-\lambda} \phi(a, c ; z)} \prec \frac{1+A z}{1+B z}
$$


and $\frac{1+A z}{1+B z}$ is the best dominant.

Proof. We get the corollary considering $q(z)=\frac{1+A z}{1+B z},-1 \leq B<A \leq 1$ in Theorem 1 .

Corollary 2. Let $\lambda>0, a, c, \alpha, \beta, \xi, \mu \in \mathbb{C}, c \neq 0,-1,-2, \ldots, \beta \neq 0$. Assume that (7) holds for $q(z)=\left(\frac{1+z}{1-z}\right)^{\gamma}, 0<\gamma \leq 1$. If

$$
\psi_{\lambda}^{a, b}(\alpha, \beta, \xi, \mu ; z) \prec \alpha+\xi\left(\frac{1+z}{1-z}\right)^{\gamma}+\mu\left(\frac{1+z}{1-z}\right)^{2 \gamma}+\frac{2 \beta \gamma z}{1-z^{2}},
$$

with $\psi_{\lambda}^{a, b}$ is defined in (8), then

$$
\frac{z\left(D_{z}^{-\lambda} \phi(a, c ; z)\right)^{\prime}}{D_{z}^{-\lambda} \phi(a, c ; z)} \prec\left(\frac{1+z}{1-z}\right)^{\gamma}
$$

and $\left(\frac{1+z}{1-z}\right)^{\gamma}$ is the best dominant.

Proof. Put $q(z)=\left(\frac{1+z}{1-z}\right)^{\gamma}, 0<\gamma \leq 1$ in Theorem 1 to obtain the corollary.

Theorem 2. Let $q$ be an analytic and univalent function in $U$ with $q(z) \neq 0, \forall z \in U$, such that $\frac{z q^{\prime}(z)}{q(z)}$ is a star-like univalent function in $U$ and

$$
\operatorname{Re}\left(\frac{2 \mu}{\beta} q^{2}(z) q^{\prime}(z)+\frac{\xi}{\beta} q(z) q^{\prime}(z)\right)>0, \text { for } \mu, \beta, \xi \in \mathbb{C}, \beta \neq 0 .
$$

If $\frac{z\left(D_{z}^{-\lambda} \phi(a, c ; z)\right)^{\prime}}{D_{z}^{-\lambda} \phi(a, c ; z)} \in Q \cap \mathcal{H}[q(0), 1]$ and $\psi_{\lambda}^{a, b}(\alpha, \beta, \xi, \mu ; z)$ is an univalent function in $U$, where $\psi_{\lambda}^{a, b}(\alpha, \beta, \xi, \mu ; z)$ is defined by (8) and $\lambda>0, a, c \in \mathbb{C}, c \neq 0,-1,-2, \ldots$, then

$$
\alpha+\xi q(z)+\mu(q(z))^{2}+\frac{\beta z q^{\prime}(z)}{q(z)} \prec \psi_{\lambda}^{a, b}(\alpha, \beta, \xi, \mu ; z)
$$

implies

$$
q(z) \prec \frac{z\left(D_{z}^{-\lambda} \phi(a, c ; z)\right)^{\prime}}{D_{z}^{-\lambda} \phi(a, c ; z)}, \quad z \in U,
$$

and function $q$ is the best subordinant.

Proof. Define the function $p$ by

$$
p(z):=\frac{z\left(D_{z}^{-\lambda} \phi(a, c ; z)\right)^{\prime}}{D_{z}^{-\lambda} \phi(a, c ; z)}, z \in U, z \neq 0 .
$$

Considering

$$
\phi(w):=\frac{\beta}{w}
$$

and

$$
v(w):=\alpha+\xi w+\mu w^{2},
$$

it is easy to show that $\phi$ is analytic in $\mathbb{C} \backslash\{0\}, \phi(w) \neq 0, w \in \mathbb{C} \backslash\{0\}$ and $v$ is also analytic in $\mathbb{C}$.

Since $\frac{v^{\prime}(q(z))}{\phi(q(z))}=\frac{q(z)[\xi+2 \mu q(z)] q^{\prime}(z)}{\beta}$, it yields

$$
\operatorname{Re}\left(\frac{v^{\prime}(q(z))}{\phi(q(z))}\right)=\operatorname{Re}\left(\frac{2 \mu}{\beta} q^{2}(z) q^{\prime}(z)+\frac{\xi}{\beta} q(z) q^{\prime}(z)\right)>0, \text { where } \mu, \beta, \alpha \in \mathbb{C}, \mu \neq 0 \text {. }
$$


From (11) and (13) we get

$$
\alpha+\xi q(z)+\mu(q(z))^{2}+\frac{\beta z q^{\prime}(z)}{q(z)} \prec \alpha+\xi p(z)+\mu(p(z))^{2}+\frac{\beta z p^{\prime}(z)}{p(z)} .
$$

Applying Lemma 2, we obtain $q(z) \prec p(z)$; therefore,

$$
q(z) \prec \frac{z\left(D_{z}^{-\lambda} \phi(a, c ; z)\right)^{\prime}}{D_{z}^{-\lambda} \phi(a, c ; z)}, \quad z \in U,
$$

and the best subordinant is function $q$.

Corollary 3. Consider $\lambda>0$ and $a, c, \alpha, \xi, \mu, \beta \in \mathbb{C}, c \neq 0,-1,-2, \ldots, \beta \neq 0$. Assume that (12) holds for $q(z)=\frac{1+A z}{1+B z},-1 \leq B<A \leq 1$. If $\frac{z\left(D_{z}^{-\lambda} \phi(a, c ; z)\right)^{\prime}}{D_{z}^{-\lambda} \phi(a, c ; z)} \in Q \cap \mathcal{H}[q(0), 1]$ and

$$
\alpha+\xi \frac{1+A z}{1+B z}+\mu\left(\frac{1+A z}{1+B z}\right)^{2}+\frac{\beta(A-B) z}{(1+A z)(1+B z)} \prec \psi_{\lambda}^{a, b}(\alpha, \beta, \xi, \mu ; z),
$$

where $\psi_{\lambda}^{a, b}$ is defined in (8), then

$$
\frac{1+A z}{1+B z} \prec \frac{z\left(D_{z}^{-\lambda} \phi(a, c ; z)\right)^{\prime}}{D_{z}^{-\lambda} \phi(a, c ; z)},
$$

and the best subordinant is $\frac{1+A z}{1+B z}$.

Proof. When $-1 \leq B<A \leq 1$, consider $q(z)=\frac{1+A z}{1+B z}$ in Theorem 2 and obtain the corollary.

Corollary 4. Let $\lambda>0, a, c, \alpha, \xi, \mu, \beta \in \mathbb{C}, c \neq 0,-1,-2, \ldots, \beta \neq 0$. Assume that (12) holds for $q(z)=\left(\frac{1+z}{1-z}\right)^{\gamma}, 0<\gamma \leq 1$. If $\frac{z\left(D_{z}^{-\lambda} \phi(a, c ; z)\right)^{\prime}}{D_{z}^{-\lambda} \phi(a, c ; z)} \in Q \cap \mathcal{H}[q(0), 1]$ and

$$
\alpha+\xi\left(\frac{1+z}{1-z}\right)^{\gamma}+\mu\left(\frac{1+z}{1-z}\right)^{2 \gamma}+\frac{2 \beta \gamma z}{1-z^{2}} \prec \psi_{\lambda}^{a, b}(\alpha, \beta, \xi, \mu ; z),
$$

where $\psi_{\lambda}^{a, b}$ is defined in (8), then

$$
\left(\frac{1+z}{1-z}\right)^{\gamma} \prec \frac{z\left(D_{z}^{-\lambda} \phi(a, c ; z)\right)^{\prime}}{D_{z}^{-\lambda} \phi(a, c ; z)}
$$

and the best subordinant is $\left(\frac{1+z}{1-z}\right)^{\gamma}$.

Proof. Put in Theorem $2 q(z)=\left(\frac{1+z}{1-z}\right)^{\gamma}$, when $0<\gamma \leq 1$.

The sandwich theorem is obtained combining Theorems 1 and 2.

Theorem 3. (Sandwich-type result) Consider $q_{1}$ and $q_{2}$ analytic and univalent functions in $U$, with $q_{1}(z) \neq 0$ and $q_{2}(z) \neq 0, \forall z \in U$, such that $\frac{z q_{1}^{\prime}(z)}{q_{1}(z)}$ and $\frac{z q_{2}^{\prime}(z)}{q_{2}(z)}$ are star-like univalent. Assume that $q_{1}$ satisfies relation (7) and $q_{2}$ satisfies relation (12). If $\frac{z\left(D_{z}^{-\lambda} \phi(a, c ; z)\right)^{\prime}}{D_{z}^{-\lambda} \phi(a, c ; z)} \in$ 
$Q \cap \mathcal{H}[q(0), 1]$ and $\psi_{\lambda}^{a, b}(\alpha, \beta, \xi, \mu ; z)$ is defined by (8) and is univalent in $U, \lambda>0$ and $a, c \in \mathbb{C}$, $c \neq 0,-1,-2, \ldots$, then $\alpha+\xi q_{1}(z)+\mu\left(q_{1}(z)\right)^{2}+\frac{\beta z q_{1}^{\prime}(z)}{q_{1}(z)} \prec \psi_{\lambda}^{a, b}(\alpha, \beta, \xi, \mu ; z) \prec \alpha+\xi q_{2}(z)+\mu\left(q_{2}(z)\right)^{2}+\frac{\beta z q_{2}^{\prime}(z)}{q_{2}(z)}$, for $\alpha, \beta, \mu, \xi \in \mathbb{C}, \beta \neq 0$, implies

$$
q_{1}(z) \prec \frac{z\left(D_{z}^{-\lambda} \phi(a, c ; z)\right)^{\prime}}{D_{z}^{-\lambda} \phi(a, c ; z)} \prec q_{2}(z),
$$

and $q_{1}$ and $q_{2}$ are, respectively, the best subordinant and the best dominant.

For $q_{1}(z)=\frac{1+A_{1} z}{1+B_{1} z}, q_{2}(z)=\frac{1+A_{2} z}{1+B_{2} z}$, where $-1 \leq B_{2}<B_{1}<A_{1}<A_{2} \leq 1$, we have the following corollary.

Corollary 5. Let $a, c, \alpha, \xi, \mu, \beta \in \mathbb{C}, c \neq 0,-1,-2, \ldots, \beta \neq 0$ and $\lambda>0$. Assume that (7) and (12) hold for $q_{1}(z)=\frac{1+A_{1} z}{1+B_{1} z}$, and $q_{2}(z)=\frac{1+A_{2} z}{1+B_{2} z},-1 \leq B_{2} \leq B_{1}<A_{1} \leq A_{2} \leq 1$. If $\frac{z\left(D_{z}^{-\lambda} \phi(a, c ; z)\right)^{\prime}}{D_{z}^{-\lambda} \phi(a, c ; z)} \in \mathcal{H}[q(0), 1] \cap Q$ and

$$
\begin{gathered}
\alpha+\xi \frac{1+A_{1} z}{1+B_{1} z}+\mu\left(\frac{1+A_{1} z}{1+B_{1} z}\right)^{2}+\frac{\beta\left(A_{1}-B_{1}\right) z}{\left(1+A_{1} z\right)\left(1+B_{1} z\right)} \prec \psi_{\lambda}^{a, b}(\alpha, \beta, \xi, \mu ; z) \\
\prec \alpha+\xi \frac{1+A_{2} z}{1+B_{2} z}+\mu\left(\frac{1+A_{2} z}{1+B_{2} z}\right)^{2}+\frac{\beta\left(A_{2}-B_{2}\right) z}{\left(1+A_{2} z\right)\left(1+B_{2} z\right)},
\end{gathered}
$$

with $\psi_{\lambda}^{a, b}$ defined by (8), then

$$
\frac{1+A_{1} z}{1+B_{1} z} \prec \frac{z\left(D_{z}^{-\lambda} \phi(a, c ; z)\right)^{\prime}}{D_{z}^{-\lambda} \phi(a, c ; z)} \prec \frac{1+A_{2} z}{1+B_{2} z},
$$

hence $\frac{1+A_{1} z}{1+B_{1} z}$ and $\frac{1+A_{2} z}{1+B_{2} z}$ are the best subordinant and the best dominant.

For $q_{1}(z)=\left(\frac{1+z}{1-z}\right)^{\gamma_{1}}, q_{2}(z)=\left(\frac{1+z}{1-z}\right)^{\gamma_{2}}$, where $0<\gamma_{1}<\gamma_{2} \leq 1$, we have the following corollary.

Corollary 6. Let $\lambda>0, a, c, \alpha, \beta, \mu, \xi \in \mathbb{C}, c \neq 0,-1,-2, \ldots, \beta \neq 0$. Assume that (7) and (12) hold for $q_{1}(z)=\left(\frac{1+z}{1-z}\right)^{\gamma_{1}}$, and $q_{2}(z)=\left(\frac{1+z}{1-z}\right)^{\gamma_{2}}, 0<\gamma_{1}<\gamma_{2} \leq 1$. If $\frac{z\left(D_{z}^{-\lambda} \phi(a, c ; z)\right)^{\prime}}{D_{z}^{-\lambda} \phi(a, c ; z)}$ $\in Q \cap \mathcal{H}[q(0), 1]$ and

$$
\begin{gathered}
\alpha+\xi\left(\frac{1+z}{1-z}\right)^{\gamma_{1}}+\mu\left(\frac{1+z}{1-z}\right)^{2 \gamma_{1}}+\frac{2 \beta \gamma_{1} z}{1-z^{2}} \prec \psi_{\lambda}^{a, b}(\alpha, \beta, \xi, \mu ; z) \\
\prec \alpha+\xi\left(\frac{1+z}{1-z}\right)^{\gamma_{2}}+\mu\left(\frac{1+z}{1-z}\right)^{2 \gamma_{2}}+\frac{2 \beta \gamma_{2} z}{1-z^{2}},
\end{gathered}
$$

where $\psi_{\lambda}^{a, b}$ is defined in (8), then

$$
\left(\frac{1+z}{1-z}\right)^{\gamma_{1}} \prec \frac{z\left(D_{z}^{-\lambda} \phi(a, c ; z)\right)^{\prime}}{D_{z}^{-\lambda} \phi(a, c ; z)} \prec\left(\frac{1+z}{1-z}\right)^{\gamma_{2}}
$$

hence $\left(\frac{1+z}{1-z}\right)^{\gamma_{1}}$ and $\left(\frac{1+z}{1-z}\right)^{\gamma_{2}}$ are the best subordinant and the best dominant, respectively. 
By changing the functions $\phi$ and $\theta$ in Theorem 1 , we get:

Theorem 4. Consider $\lambda>0, a, c \in \mathbb{C}, c \neq 0,-1,-2, \ldots$, the convex and univalent function $q$ in $U$ with $q(0)=\lambda$ and $\frac{z\left(D_{z}^{-\lambda} \phi(a, c ; z)\right)^{\prime}}{D_{z}^{-\lambda} \phi(a, c ; z)} \in \mathcal{H}(U), \forall z \in U$. Suppose that

$$
\operatorname{Re}\left(z \frac{q^{\prime \prime}(z)}{q^{\prime}(z)}+\frac{\alpha}{\beta}+1\right)>0, \quad z \in U
$$

where $\alpha, \beta \in \mathbb{C}, \beta \neq 0$, and

$$
\begin{gathered}
\psi_{\lambda}^{a, b}(\alpha, \beta ; z):=(\alpha+\beta) \frac{z\left(D_{z}^{-\lambda} \phi(a, c ; z)\right)^{\prime}}{D_{z}^{-\lambda} \phi(a, c ; z)}-\beta\left(\frac{z\left(D_{z}^{-\lambda} \phi(a, c ; z)\right)^{\prime}}{D_{z}^{-\lambda} \phi(a, c ; z)}\right)^{2} \\
+\beta \frac{z^{2}\left(D_{z}^{-\lambda} \phi(a, c ; z)\right)^{\prime \prime}}{D_{z}^{-\lambda} \phi(a, c ; z)} .
\end{gathered}
$$

If the following subordination is satisfied by $q$,

$$
\psi_{\lambda}^{a, b}(\alpha, \beta ; z) \prec \alpha q(z)+\beta z q^{\prime}(z), \quad z \in U,
$$

then

$$
\frac{z\left(D_{z}^{-\lambda} \phi(a, c ; z)\right)^{\prime}}{D_{z}^{-\lambda} \phi(a, c ; z)} \prec q(z), \quad z \in U,
$$

and the best dominant is the function $q$.

Proof. Define

$$
p(z):=\frac{z\left(D_{z}^{-\lambda} \phi(a, c ; z)\right)^{\prime}}{D_{z}^{-\lambda} \phi(a, c ; z)}, z \in U, z \neq 0
$$

with $p(0)=\lambda$, an analytic function in $U$. Differentiating with respect to $z$ we get $p^{\prime}(z)=$ $\frac{\left(D_{z}^{-\lambda} \phi(a, c ; z)\right)^{\prime}}{D_{z}^{-\lambda} \phi(a, c ; z)}-z\left(\frac{\left(D_{z}^{-\lambda} \phi(a, c ; z)\right)^{\prime}}{D_{z}^{-\lambda} \phi(a, c ; z)}\right)^{2}+z \frac{\left(D_{z}^{-\lambda} \phi(a, c ; z)\right)^{\prime \prime}}{D_{z}^{-\lambda} \phi(a, c ; z)}$ and

$$
z p^{\prime}(z)=\frac{z\left(D_{z}^{-\lambda} \phi(a, c ; z)\right)^{\prime}}{D_{z}^{-\lambda} \phi(a, c ; z)}-\left(\frac{z\left(D_{z}^{-\lambda} \phi(a, c ; z)\right)^{\prime}}{D_{z}^{-\lambda} \phi(a, c ; z)}\right)^{2}+\frac{z^{2}\left(D_{z}^{-\lambda} \phi(a, c ; z)\right)^{\prime \prime}}{D_{z}^{-\lambda} \phi(a, c ; z)} .
$$

Let

$$
\phi(w):=\beta
$$

be analytic in $\mathbb{C} \backslash\{0\}$ with $\phi(w) \neq 0, w \in \mathbb{C} \backslash\{0\}$ and

$$
\theta(w):=\alpha w
$$

analytic in $\mathbb{C}$.

Consider

$$
Q(z)=z q^{\prime}(z) \phi(q(z))=\beta z q^{\prime}(z)
$$

star-like univalent in $U$ and

$$
h(z)=\theta(q(z))+Q(z)=\alpha q(z)+\beta z q^{\prime}(z) .
$$

We obtain $\operatorname{Re}\left(\frac{z h^{\prime}(z)}{Q(z)}\right)=\operatorname{Re}\left(z \frac{q^{\prime \prime}(z)}{q^{\prime}(z)}+\frac{\alpha}{\beta}+1\right)>0$. 
From (19), we get $\alpha p(z)+\beta z p^{\prime}(z)=(\alpha+\beta) \frac{z\left(D_{z}^{-\lambda} \phi(a, c ; z)\right)^{\prime}}{D_{z}^{-\lambda} \phi(a, c ; z)}-\beta\left(\frac{z\left(D_{z}^{-\lambda} \phi(a, c ; z)\right)^{\prime}}{D_{z}^{-\lambda} \phi(a, c ; z)}\right)^{2}+$ $\beta \frac{z^{2}\left(D_{z}^{-\lambda} \phi(a, c ; z)\right)^{\prime \prime}}{D_{z}^{-\lambda} \phi(a, c ; z)}$.

By using (17), we get $\alpha p(z)+\beta z p^{\prime}(z) \prec \alpha q(z)+\beta z q^{\prime}(z)$.

Lemma 1 gives $p(z) \prec q(z), \forall z \in U$, so we obtain $\frac{z\left(D_{z}^{-\lambda} \phi(a, c ; z)\right)^{\prime}}{D_{z}^{-\lambda} \phi(a, c ; z)} \prec q(z), \forall z \in U$, and the best dominant is function $q$.

Corollary 7. Let $-1 \leq B<A \leq 1, \lambda>0, a, c, \alpha, \beta \in \mathbb{C}, c \neq 0,-1,-2, \ldots, \beta \neq 0$, and $q(z)=\frac{1+A z}{1+B z}, z \in U$, fulfilling the relation (15). If

$$
\psi_{\lambda}^{a, b}(\alpha, \beta ; z) \prec \frac{\beta(A-B) z}{(1+B z)^{2}}+\alpha \frac{1+A z}{1+B z},
$$

with $\psi_{\lambda}^{a, b}$ defined by (16), then

$$
\frac{z\left(D_{z}^{-\lambda} \phi(a, c ; z)\right)^{\prime}}{D_{z}^{-\lambda} \phi(a, c ; z)} \prec \frac{1+A z}{1+B z}
$$

and the best dominant is $\frac{1+A z}{1+B z}$.

Proof. In Theorem 4 consider $q(z)=\frac{1+A z}{1+B z}$, with $-1 \leq B<A \leq 1$.

Corollary 8. Let $\lambda>0, a, c, \alpha, \beta \in \mathbb{C}, c \neq 0,-1,-2, \ldots, \beta \neq 0$ and $q(z)=\left(\frac{1+z}{1-z}\right)^{\gamma}$, $0<\gamma \leq 1$, fulfilling the relation (15). If

$$
\psi_{\lambda}^{a, b}(\alpha, \beta ; z) \prec \frac{2 \beta \gamma z}{1-z^{2}}\left(\frac{1+z}{1-z}\right)^{\gamma}+\alpha\left(\frac{1+z}{1-z}\right)^{\gamma}
$$

with $\psi_{\lambda}^{a, b}$ defined by (16), then

$$
\frac{z\left(D_{z}^{-\lambda} \phi(a, c ; z)\right)^{\prime}}{D_{z}^{-\lambda} \phi(a, c ; z)} \prec\left(\frac{1+z}{1-z}\right)^{\gamma}
$$

and $\left(\frac{1+z}{1-z}\right)^{\gamma}$ is the best dominant.

Proof. In Theorem 4 put $q(z)=\left(\frac{1+z}{1-z}\right)^{\gamma}$, with $0<\gamma \leq 1$.

By changing the functions $\phi$ and $\theta$ in Theorem 3 to be the same as in Theorem 4 , we get:

Theorem 5. Let $\lambda>0, a, c \in \mathbb{C}, c \neq 0,-1,-2, \ldots$ and $q$ be a convex and univalent function in $U$ with $q(0)=\lambda$. Suppose that

$$
\operatorname{Re}\left(\frac{\alpha}{\beta} q^{\prime}(z)\right)>0, \alpha, \beta \in \mathbb{C}, \beta \neq 0
$$

If $\frac{z\left(D_{z}^{-\lambda} \phi(a, c ; z)\right)^{\prime}}{D_{z}^{-\lambda} \phi(a, c ; z)} \in Q \cap \mathcal{H}[q(0), 1]$ and $\psi_{\lambda}^{a, b}(\alpha, \beta ; z)$ defined in (16) is univalent in $U$, then

$$
\alpha q(z)+\beta z q^{\prime}(z) \prec \psi_{\lambda}^{a, b}(\alpha, \beta ; z)
$$


implies

$$
q(z) \prec \frac{z\left(D_{z}^{-\lambda} \phi(a, c ; z)\right)^{\prime}}{D_{z}^{-\lambda} \phi(a, c ; z)}, \forall z \in U,
$$

and the best subordinant is the function $q$.

Proof. Define the analytic function

$$
p(z):=\frac{z\left(D_{z}^{-\lambda} \phi(a, c ; z)\right)^{\prime}}{D_{z}^{-\lambda} \phi(a, c ; z)}, z \in U, z \neq 0
$$

and $p(0)=\lambda$.

Set

$$
\phi(w):=\beta
$$

to be analytic in $\mathbb{C} \backslash\{0\}$ with $\phi(w) \neq 0, \forall w \in \mathbb{C} \backslash\{0\}$ and

$$
v(w):=\alpha w
$$

analytic in $\mathbb{C}$ $\beta \neq 0$.

Since $\frac{v^{\prime}(q(z))}{\phi(q(z))}=\frac{\alpha}{\beta} q^{\prime}(z)$, from $(20)$ we get $\operatorname{Re}\left(\frac{v^{\prime}(q(z))}{\phi(q(z))}\right)=\operatorname{Re}\left(\frac{\alpha}{\beta} q^{\prime}(z)\right)>0$, with $\alpha, \beta \in \mathbb{C}$,

Relation (21) gives the differential superordination

$$
\alpha q(z)+\beta z q^{\prime}(z) \prec \alpha p(z)+\beta z p^{\prime}(z), \quad z \in U,
$$

and by applying Lemma 2, we obtain $q(z) \prec p(z)$, which means

$$
q(z) \prec \frac{z\left(D_{z}^{-\lambda} \phi(a, c ; z)\right)^{\prime}}{D_{z}^{-\lambda} \phi(a, c ; z)}, \quad z \in U,
$$

and the best subordinant is the function $q$.

Corollary 9. Assume that (20) holds for $q(z)=\frac{1+A z}{1+B z}, z \in U$, with $-1 \leq B<A \leq 1, \lambda>0, a$, $c \in \mathbb{C}, c \neq 0,-1,-2, \ldots$. If $\frac{z\left(D_{z}^{-\lambda} \phi(a, c ; z)\right)^{\prime}}{D_{z}^{-\lambda} \phi(a, c ; z)} \in Q \cap \mathcal{H}[q(0), 1]$, and

$$
\alpha \frac{1+A z}{1+B z}+\frac{\beta(A-B) z}{(1+B z)^{2}} \prec \psi_{\lambda}^{a, b}(\alpha, \beta ; z),
$$

where $\psi_{\lambda}^{a, b}$ is defined in (16), $\alpha, \beta \in \mathbb{C}, \beta \neq 0$, then

$$
\frac{1+A z}{1+B z} \prec \frac{z\left(D_{z}^{-\lambda} \phi(a, c ; z)\right)^{\prime}}{D_{z}^{-\lambda} \phi(a, c ; z)},
$$

and the best subordinant is $\frac{1+A z}{1+B z}$.

Proof. In Theorem 5 consider $q(z)=\frac{1+A z}{1+B z}$, with $-1 \leq B<A \leq 1$.

Corollary 10. Assume that (20) holds for $q(z)=\left(\frac{1+z}{1-z}\right)^{\gamma}, 0<\gamma \leq 1, \lambda>0, a, c \in \mathbb{C}$, $c \neq 0,-1,-2, \ldots$ If $\frac{z\left(D_{z}^{-\lambda} \phi(a, c ; z)\right)^{\prime}}{D_{z}^{-\lambda} \phi(a, c ; z)} \in Q \cap \mathcal{H}[q(0), 1]$ and

$$
\alpha\left(\frac{1+z}{1-z}\right)^{\gamma}+\frac{2 \beta \gamma z}{1-z^{2}}\left(\frac{1+z}{1-z}\right)^{\gamma} \prec \psi_{\lambda}^{a, b}(\alpha, \beta ; z),
$$


where $\psi_{\lambda}^{a, b}$ is defined in (16), $\alpha, \beta \in \mathbb{C}$ and $\beta \neq 0$, then

$$
\left(\frac{1+z}{1-z}\right)^{\gamma} \prec \frac{z\left(D_{z}^{-\lambda} \phi(a, c ; z)\right)^{\prime}}{D_{z}^{-\lambda} \phi(a, c ; z)},
$$

and the best subordinant is $\left(\frac{1+z}{1-z}\right)^{\gamma}$.

Proof. In Theorem 5 consider $q(z)=\left(\frac{1+z}{1-z}\right)^{\gamma}$, with $0<\gamma \leq 1$.

The sandwich theorem is obtained combining Theorem 4 and Theorem 5 .

Theorem 6. (Sandwich-type result) Consider $q_{1}$ and $q_{2}$ convex and univalent functions in $U$ with $q_{1}(z) \neq 0$ and $q_{2}(z) \neq 0, \forall z \in U$. Assume that relation (15) is satisfied by $q_{1}$ and relation (20) is satisfied by $q_{2}$. If $\frac{z\left(D_{z}^{-\lambda} \phi(a, c ; z)\right)^{\prime}}{D_{z}^{-\lambda} \phi(a, c ; z)} \in Q \cap \mathcal{H}[q(0), 1]$ and $\psi_{\lambda}^{a, b}(\alpha, \beta ; z)$ is univalent in $U$ defined by (16), $\lambda>0, a, c, \alpha, \beta \in \mathbb{C}, c \neq 0,-1,-2, \ldots, \beta \neq 0$, then

$$
\alpha q_{1}(z)+\beta z q_{1}^{\prime}(z) \prec \psi_{\lambda}^{a, b}(\alpha, \beta ; z) \prec \alpha q_{2}(z)+\beta z q_{2}^{\prime}(z),
$$

implies

$$
q_{1}(z) \prec \frac{z\left(D_{z}^{-\lambda} \phi(a, c ; z)\right)^{\prime}}{D_{z}^{-\lambda} \phi(a, c ; z)} \prec q_{2}(z), \forall z \in U,
$$

and the best subordinant is $q_{1}$ and the best dominant is $q_{2}$.

Letting $q_{1}(z)=\frac{1+A_{1} z}{1+B_{1} z}, q_{2}(z)=\frac{1+A_{2} z}{1+B_{2} z}$, where $-1 \leq B_{2}<B_{1}<A_{1}<A_{2} \leq 1$, in Theorem 6 we get

Corollary 11. Assume that (15) and (20) hold for $q_{1}(z)=\frac{1+A_{1} z}{1+B_{1} z}$ and $q_{2}(z)=\frac{1+A_{2} z}{1+B_{2} z}$, and $\lambda>0, a, c, \alpha, \beta \in \mathbb{C}, c \neq 0,-1,-2, \ldots, \beta \neq 0,-1 \leq B_{2} \leq B_{1}<A_{1} \leq A_{2} \leq 1$. If $\frac{z\left(D_{z}^{-\lambda} \phi(a, c ; z)\right)^{\prime}}{D_{z}^{-\lambda} \phi(a, c ; z)} \in Q \cap \mathcal{H}[q(0), 1]$ and

$$
\begin{aligned}
& \alpha \frac{1+A_{1} z}{1+B_{1} z}+\frac{\beta\left(A_{1}-B_{1}\right) z}{\left(1+B_{1} z\right)^{2}} \prec \psi_{\lambda}^{a, b}(\alpha, \beta ; z) \\
& \prec \alpha \frac{1+A_{2} z}{1+B_{2} z}+\frac{\beta\left(A_{2}-B_{2}\right) z}{\left(1+B_{2} z\right)^{2}}, \forall z \in U,
\end{aligned}
$$

where $\psi_{\lambda}^{a, b}$ is defined in (16), $\alpha, \beta \in \mathbb{C}$ and $\beta \neq 0$, then

$$
\frac{1+A_{1} z}{1+B_{1} z} \prec \frac{z\left(D_{z}^{-\lambda} \phi(a, c ; z)\right)^{\prime}}{D_{z}^{-\lambda} \phi(a, c ; z)} \prec \frac{1+A_{2} z}{1+B_{2} z}, \quad z \in U ;
$$

hence, the best subordinant is $\frac{1+A_{1} z}{1+B_{1} z}$ and the best dominant is $\frac{1+A_{2} z}{1+B_{2} z}$. we obtain

By setting $q_{1}(z)=\left(\frac{1+z}{1-z}\right)^{\gamma_{1}}$ and $q_{2}(z)=\left(\frac{1+z}{1-z}\right)^{\gamma_{2}}$, where $0<\gamma_{1}<\gamma_{2} \leq 1$, in Theorem 6 
Corollary 12. Assume that (15) and (20) hold for $q_{1}(z)=\left(\frac{1+z}{1-z}\right)^{\gamma_{1}}$ and $q_{2}(z)=\left(\frac{1+z}{1-z}\right)^{\gamma_{2}}$, and $\lambda>0, a, c, \alpha, \beta \in \mathbb{C}, c \neq 0,-1,-2, \ldots, \beta \neq 0,0<\gamma_{1}<\gamma_{2} \leq 1$. If $\frac{z\left(D_{z}^{-\lambda} \phi(a, c ; z)\right)^{\prime}}{D_{z}^{-\lambda} \phi(a, c ; z)} \in$ $Q \cap \mathcal{H}[q(0), 1]$ and

$$
\begin{gathered}
\alpha\left(\frac{1+z}{1-z}\right)^{\gamma_{1}}+\frac{2 \beta \gamma_{1} z}{1-z^{2}}\left(\frac{1+z}{1-z}\right)^{\gamma_{1}} \prec \psi_{\lambda}^{a, b}(\alpha, \beta ; z) \\
\prec \alpha\left(\frac{1+z}{1-z}\right)^{\gamma_{2}}+\frac{2 \beta \gamma_{2} z}{1-z^{2}}\left(\frac{1+z}{1-z}\right)^{\gamma_{2}}, \quad z \in U,
\end{gathered}
$$

where $\psi_{\lambda}^{a, b}$ is defined by (16), then

$$
\left(\frac{1+z}{1-z}\right)^{\gamma_{1}} \prec \frac{z\left(D_{z}^{-\lambda} \phi(a, c ; z)\right)^{\prime}}{D_{z}^{-\lambda} \phi(a, c ; z)} \prec\left(\frac{1+z}{1-z}\right)^{\gamma_{2}}, \forall z \in U ;
$$

hence, the best subordinant is $\left(\frac{1+z}{1-z}\right)^{\gamma_{1}}$ and the best dominant is $\left(\frac{1+z}{1-z}\right)^{\gamma_{2}}$.

\section{Discussion}

Using the previously introduced operator involving the fractional integral of confluent hypergeometric function, further study was done and new subordinations and superordinations were obtained; we also gave their best subordinants and best dominants. Interesting corollaries were stated using particular functions as best subordinants and best dominants of the subordinations and superordinations studied in the theorems stated in this paper. An investigation on this operators' univalence is yet to be done. Additionally, other aspects related to it can still be investigated, such as introducing new classes of functions with certain properties given by the use of this operator.

Funding: This research received no external funding.

Institutional Review Board Statement: Not applicable.

Informed Consent Statement: Not applicable.

Data Availability Statement: Not applicable.

Acknowledgments: Dedicated to memory of Mitrofan Cioban.

Conflicts of Interest: The author declares no conflict of interest.

\section{References}

1. Alb Lupaş, A. Subordination results for fractional integral associated with Dziok-Srivastava operator. An. Univ. Oradea Fasc. Math. 2020, 27, 125-131.

2. Alb Lupaş, A.; Cioban, M. On a subclass of analytic functions defined by a fractional integral operator. In Proceedings of the International Conference on Mathematics, Informatics and Information Technologies, Bălți, Moldova, 19-21 April 2018; pp. 8-14.

3. Owa, S. On the distortion theorems I. Kyungpook Math. J. 1978, 18, 53-59.

4. Viglialoro, G.; Murcia, J. A singular elliptic problem related to the membrane equilibrium equations. Int. J. Comput. Math. 2013, 90, 2185-2196. [CrossRef]

5. Li, T.; Pintus, N.; Viglialoro, G. Properties of solutions to porous medium problems with different sources and boundary conditions. Z. Angew. Math. Phys. 2019, 70,1-18. [CrossRef]

6. Li, T.; Viglialoro, G. Analysis and explicit solvability of degenerate tensorial problems. Bound. Value Probl. 2018, 2, 1-13. [CrossRef]

7. Alb-Lupaş, A.; Oros, G.I. Differential subordination and superordination results using fractional integral of confluent hypergeometric function. Symmetry 2021, 13, 327. [CrossRef]

8. Chaudhry, M.A.; Qadir, A.; Srivastava, H.M. Extended hypergeometric and confluent hypergeometric functions. Appl. Math. Comput. 2004, 159, 589-602. [CrossRef]

9. Ghanim, F.; Al-Shaqsi, K.; Darus, M.; Al-Janaby, H.F. Subordination Properties of Meromorphic Kummer Function Correlated with Hurwitz-Lerch Zeta-Function. Mathematics 2021, 9, 192. [CrossRef]

10. Oros, G.I. New Conditions for Univalence of Confluent Hypergeometric Function. Symmetry 2021, 13, 82. [CrossRef] 
11. Kummer, E.E. De integralibus quibusdam definitis et seriebus infinitis. J. für die reine und angewandte. Mathematik 1837, 17, 228-242. (In Latin)

12. Miller, S.S.; Mocanu, P.T. Differential Subordinations. In Theory and Applications; Marcel Dekker Inc.: New York, NY, USA; Basel, Switzerland, 2000.

13. Miller, S.S.; Mocanu, P.T. Subordinants of differential superordinations. Complex Var. 2003, 48, 815-826. [CrossRef]

14. Bulboacă, T. Classes of first order differential superordinations. Demonstr. Math. 2002, 35, 287-292. [CrossRef] 